makes the water very alkaline so an acid has to be added to bring the $\mathrm{pH}$ to the acceptable level of $7 \cdot 2-8 \cdot 0$. If the $\mathrm{pH}$ is right and there is free chlorine in the pool, it is very unlikely that problems will ever be encountered.

HARVEY GORDON Surrey Area Health Authority,
Mid-Surrey District,
West Park Hospital,

West Park

\section{Abortion and maternal deaths}

SIR,-Mr Caspar Brook (28 August, p 524) correctly quotes our study as having shown that a majority of women seeking therapeutic abortion contact the health services at an early stage in pregnancy and suffer delays thereafter. ${ }^{1}$ These findings were subsequently supported by others. ${ }^{2-4}$ However, he did not make it clear that our study related to a period before the University Hospital of Wales was opened and before any members of the current professorial team had arrived in Cardiff.

$\mathrm{Mr}$ Brook touches on another point (help with terminations) which is perhaps best illustrated by a quotation from a report on Welsh patients in 1975 produced by the British Pregnancy Advisory Service: "Overall, Welsh women are more likely to get NHS abortions than English women unless they live in Cardiff. The differences are so great between Cardiff and Swansea that they cannot be accounted for in medical resource terms only." Like other local variations, this is a reflection of the prevailing moral positions of gynaecologists in their interpretation of the Abortion Act.

Surely we have already tolerated for too long the dual iniquities whereby on the one hand gynaecologists with sincerely held convictions have to resist pressure to interpret the Act more liberally ${ }^{5}$ and on the other hand a woman's address is the most important determinant of whether or not she will have to pay for an abortion. Dr Selwyn Crawford ${ }^{6}$ and others ${ }^{7}$ have suggested that a way out of this dilemma is to remove work involving the termination of fetal life from mainstream gynaecology. This is the way the non-profit-making private sector has overcome the problem. When are we going to see a similar development within the NHS ?

IAIN Chalmers

\section{W Ish National School of Medicine,}

Cardiff

Chalmers, I, and Anderson, A B M, Lancet, 1972, 1 , 1324 .

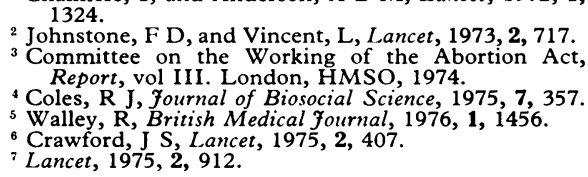

\section{Teaching teaching}

SIR,-Dr D P Mason's Personal View (14 August, $p$ 418) reveals that he has learnt much from his first year as a trainer and indeed, by his side-swipes at academics, reveals that he has also learnt much about learning (equals teaching) by reading and by contacts with others.

Teachers in general practice are taking on this task as a second professional commitment. ${ }^{1}$ Many teaching skills are practised by all general practitioners in the very nature of their work. Extra skills are desirable in teachers and, while a talented few may have these intuitively, most of us need to acquire and practise them with our peers.

Courses for teachers are organised in such a way that participants learn mostly from each other and not from "educationalist peers." Perhaps Dr Mason would contribute to a teacher course more than he would gain from the experience of it, but would that be a good reason for abstention?

D J PRICE

Postgraduate Adviser in General Practice,

London NW1

${ }^{1}$ Byrne, P S, and Long, B E L, Learning to Care.
Edinburgh and London, Churchill Livingstone, Edinb 1973.

\section{Postural effect of diazoxide}

SIR,-The management of severe hypertension has been improved considerably by the introduction of intravenous diazoxide. The standard therapy is $300 \mathrm{mg}$ given by fast injection, though the need for rapidity has been challenged. The response is generally prompt and may be sustained long enough to permit maintenance therapy to be instituted after a single dose. Failure of effect may lead to repeating the same dose, doubling it, or using alternative agents. If the speed of the first injection was slow then rapid repeat injection is recommended by the manufacturers. This case report suggests that posture may be important in the response to diazoxide.

A 53-year-old woman developed a sudden severe headache. Her own doctor prescribed chlorthalidone for this after finding her blood pressure to be $160 / 120 \mathrm{~mm} \mathrm{Hg}$. Her symptom improved, but she again developed a severe occipital headache after three weeks. On admission to hospital her blood pressure was $230 / 130 \mathrm{~mm} \mathrm{Hg}$. Her retinae showed arterial narrowing, haemorrhages, and blurred margins to the nasal half of both optic discs. She promptly had a generalised convulsion, which was controlled by diazepam $10 \mathrm{mg}$ intramuscularly. An intravenous injection of $300 \mathrm{mg}$ of diazoxide was given as quickly as possible, with an ill sustained moderate fall in blood pressure to $190 / 110$ $\mathrm{mm} \mathrm{Hg}$. After four hours, when the blood pressure had reached $210 / 125 \mathrm{~mm} \mathrm{Hg}$, a repeat injection was given, in approximately $1 \frac{1}{2}$ minutes, with a temporary marked fall in blood pressure to $130 / 70$ $\mathrm{mm} \mathrm{Hg}$. After a further three hours a further injection of diazoxide was considered since the blood pressure had risen to $250 / 140 \mathrm{~mm} \mathrm{Hg}$, but before this was given the bed was tilted $10^{\circ}$ foot down. A satisfactory fall in blood pressure to a mean level of $170 / 95 \mathrm{~mm} \mathrm{Hg}$ occurred and this was sustained for 10 hours. It was then possible to start therapy with oral bethanidine. Blood sugar was monitored and did not exceed $9.4 \mathrm{mmol} / \mathrm{l}$ (170 $\mathrm{mg} / 100 \mathrm{ml}$ ). Blood urea was normal.

The patient was stuporous throughout. There was intermittent right facial weakness, hyperreflexia in the right arm was noted, and the lef plantar response was extensor. A lumbar puncture revealed uniformly bloodstained cerebrospinal fluid with a pressure of $320 \mathrm{~mm}$ fluid. The ches radiograph showed a normal heart size and the electrocardiogram marginal left ventricular hypertrophy. Angiography subsequently showed an aneurysm of the left posterior communicating artery. During and after neurosurgery the antihypertensive therapy had to be temporarily withdrawn and the blood pressure rose again to a persistent level of $180 / 120 \mathrm{~mm} \mathrm{Hg}$.

Diazoxide has been approved in Britain for use in hypertensive crises for several years now. A number of articles have appeared on its use. None of them mention the possibility of tilting the bed to potentiate the effect, though postural hypotension is mentioned as an uncommon side effect. Apparently this refers only to vertical posture. The onset of effect is agreed to be within a matter of minutes. There is a wide range of opinion about the duration of action, variously stated as 2-6 hours, ${ }^{1} 1-18$ hours, ${ }^{2} 3-8$ hours, ${ }^{3}$ and 4-24 hours. ${ }^{4}$ Frusemide is claimed to combat the fluid-retaining effect and thus potentiate the hypotensive action. ${ }^{2}$

In the case reported here there was an unsatisfactory response to intravenous diazoxide on two occasions. The blood pressure had risen alarmingly only $2 \frac{1}{2}$ hours after the second injection and the question arose of repeating diazoxide or using alternative agents. After bed tilting these became unnecessary. This suggests that the effect of the diazoxide was potentiated. It seems very unlikely that this manoeuvre itself could have controlled such severe hypertension. In view of the subsequent relapse of hypertension after surgery and withdrawal of specific therapy spontaneous remission cannot reasonably be claimed to account for the effects noted.

However, severe hypertension may be labile and the response to diazoxide ill-defined in amount and duration. The correct timing of repeat injections is a matter of individual judgment. The findings reported indicate that lack of response may be remedied by tilting the patient's bed before further therapy is considered. It could be that this ought to be an integral part of diazoxide therapy from the start in all but comatose patients likely to inhale vomit.

Professor I A D Bouchier kindly gave permission for this case to be reported.

M C BATESON

University Department of Medicine,

Ninewells Hospital,

Dundee

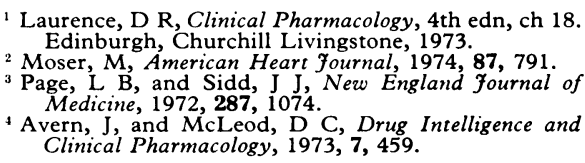

Risk of haemorrhage in oxytocin stress test

SIR,-Though the oxytocin stress test has proved to be of considerable value in antenatal monitoring, ${ }^{12}$ it could be dangerous in patients with placenta praevia, as illustrated by the following case.

A 31-year-old Malay woman, gravida 6, para 3 , was admitted with suspected intrauterine growth retardation. She was uncertain of her menstrual history. There was no relevant obstetric history and no predisposing causes were elicited to account for the intrauterine growth retardation. The positive findings were a lax abdomen with a single fetus in breech presentation. The oxytocin stress test as described by Weingold $e t a l^{2}$ was performed on two occasions separated by an interval of three days. On both occasions the tracings showed a normal pattern. However, a day after the second test the patient began to have intermittent vaginal bleeding not associated with any apparent contractions. An immediate placental scan with radioactive indium showed the presence of a major placenta praevia. This was confirmed subsequently at examination under anaesthesia and lower-segment caesarean section. A baby boy of $3000 \mathrm{~g}$ was safely delivered. The baby did not show features of intrauterine growth retardation.

Oxytocin stress tests and antenatal cardiotocography are becoming more commonly used to diagnose placental insufficiency in many 
hospitals. In some hospitals induction for growth retardation is performed only after antenatal cardiotocography has shown some abnormality of the fetal heart rate. ${ }^{3}$ As the oxytocin stress test becomes more routine, the risks of inadvertent induction of antepartum haemorrhage in unsuspected cases of placenta praevia increase. In view of the danger of producing haemorrhage it would be advantageous not to perform an oxytocin stress tes on patients in whom placenta praevia is suspected.

$\mathrm{K} \mathrm{H} \mathrm{NG}$

\section{Department of Obstetrics and Gynaecology,}

University Hospital

Kuala Lumpur, Malaysi

Emmen, L, et al, British fournal of Obstetrics and Gynaecology, 1975, 82, 353 .

Weingold, A B, et al, American fournal of Obstetrics and Gynecology, 1975, 123, 466

immons, S, in Proceedings of Third Study Group of the Royal College of Obstetricians and Gynaecologists,

\section{Raynaud's disease and oral contraceptives}

SIR,-The recent correspondence on this subject (17 July, p 156; 21 August, p 477) omits to mention the fact that patients with Raynaud's syndrome, either of an idiopathic nature or due to scleroderma, have raised plasma fibrinogen levels. ${ }^{2}$ Work in our laboratories (to be published) has confirmed previous workers' findings ${ }^{3}$ that the use of the contraceptive pill causes a significant elevation of plasma fibrinogen values. As raised fibrinogen levels increase whole-blood viscosity ${ }^{4}$ it would seem rational to encourage patients with Raynaud's syndrome not to take the contraceptive pill.

We have followed this policy in three cases of Raynaud's syndrome and have found that these patients, on ceasing to take the pill, have had a fall in fibrinogen levels and a noticeable symptomatic improvement. One patient was unwilling to change her form of contraception but as progesterones have been shown not to increase fibrinogen levels ${ }^{5}$ her prescription was changed from a combined oestrogen/progesterone pill to a progesterone pill. After this her fibrinogen level fell and her hands felt warmer.

I would suggest that Raynaud's syndrome, whether of idiopathic causation or due to scleroderma, is indeed a contraindication to taking the combined oestrogen/progesterone contraceptive pill.

P E M JARRETT

Department of Surgery, St Thomas's Hospital, London SEl

1 Jarrett, P E M, et al, British fournal of Surgery, 1975, 8, 62.

Pringle, R, Walder, D N, and Weaver, J P A, Lancet, $1965,1,1086$.

${ }^{3}$ Brackman, P, and Astrup, T, Lancet, 1964, 2, 10.

Weaver, J P A, Evans, A, and Walder, D N, Clinical

Poller, L, et al, British Medical fournal, 1969, 1, 554.

\section{Space phobia and the " $3 F$ syndrome"}

SIR,-Could Drs I Marks and P Bebbington (7 August, p 345) be talking about a condition well known to physicians in geriatric medicine which I have called the " $3 \mathrm{~F}$ syndrome" (fear of further falling) ${ }^{1}$ and which is extremely common in elderly women? Incidentally, by elderly I mean women over the age of 75 ; the average age of Drs Marks and Bebbington's patients was 56.5 years.

Patients who have in the past experienced a fall become extremely frightened and anxious and I have always attributed this to the shock effect of discovering that their body, which has formerly behaved in a stable and predictable fashion, now unpredictably and literally "lets them down." The syndrome has two clinical accompaniments: the " $3 \mathrm{G}$ syndrome" and the "3P syndrome." The former means "grabbing great-grandmother" (a further indication of the usual age distribution), while the latter refers to the "patient with precocious parking." The former term describes the apparently irresistible need of patients with this syndrome to clutch or grab on to any object, without regard to its value as a source of support or to its proximity, while the latter reminds us that these patients make major errors of judgmen when approaching a chair and tend to reach for it in an attempt to sit down long before this is appropriate.

Other features found in association with the " $3 \mathrm{~F}$ syndrome" include: a tendency to stand with the arms extended, abducted, and supinated, which is very reminiscent of the Moro reflex in infants; a disturbance of the flexion synergy on rising from a chair, which is often replaced by a tendency to extend the arms. legs, and trunk and force the patien back into the chair; and the habit of repeatedly uttering the phrase "I am going to fall" when in a state of stability. Many of these symptoms are rapidly reversed by rehabilitation or by the provision of minimal support, and it seems as though the disturbance of visuospatial perception and movement pattern is aggravated by anxiety.

My colleagues and I are about to embark on a research project into falls in old age, in which among other things, we will be attempting to relate this clinical picture to the patient's personality and to the pathological condition responsible for the falls.

BERNARD ISAAC

University Department of Geriatric

Medicine,
Queen Elizabeth Hospital,

Birmingham

Isaacs, B, The Giants of Geriatrics, Inaugural Lecture University of Birmingham, 1976.

\section{"The Forbidden Love"}

SIR,-The patronising and dismissive allusion to what he supposes are the conclusions of the authors of "The Forbidden Love" which Dr Sydney Brandon makes in his review (28 August, $p$ 533) hopelessly miss the point. There is no doubt from the text that they clearly mark off "care and respect" for children ( $p$ 89) as well as non-deviant unpleasantness to them (p 93), from deviant and manifestly erotic paedophilia, which was unequivocally seen as destructive and dangerous ( $p$ 127) as well as pathological (p 100).

So puzzled was I by the total lack of resemb lance between the book and Dr Brandon's review of it that I was left wondering whether he could have read it at all; or if he had, whether it was with powerful prejudice; or otherwise whether he could have the necessary theoretical equipment to assess it critically. How otherwise could he fail to see the authors' intention to demonstrate the mechanisms that underlie paedophilia and the ways in which these may on the one hand be incorporated in a manner that is beneficial personally (p 92) and ethologically ( $\mathrm{p}$ 37) or on the other become perverted and so give rise to deviant behaviour (for example, pp 99-100)?

It is a pity that such a valuable study could not have received a less trifling notice which would have done justice to its merit as well as to the real, rather than fancied dismerits-its lurid cover, its sensationalist title, and its lack of a bibliography, for instance.

R Carvalho

Department of Child Psychiatry, St George's Hospital,
London SW 17

\section{Antibiotics for sore throats}

SIR,-I was rather surprised by your expert's answer (19 June, p 1516) to the question of withholding antibiotics when treating sore throats. Is it now in fact established paediatric practice "to give oral penicillin at least to children because $(a)$ it does no harm and $(b)$ rheumatic fever and acute nephritis are occasionally seen, though increasingly rarely ?"

J N STENHOUSE

Farringdon,

Berks

\section{Folate deficiency and neurological} disease

SIR,-Drs M Manzoor and J Runcie are to be commended for their simple and clear data presentation (15 May, p 1176). I would like to make some comments on their observations.

(1) In a paper dated January $1976^{1}$ no referred to by the authors I described four women aged between 32 and 44 who had folate deficiency, signs of polyneuropathy, vibration sense impairment, mild depression, and subtotal villous atrophy in the jejunal mucosa. Only one patient had megaloblastosis, but two had low cerebrospinal fluid (CSF) folate levels.

(2) From all our studies ${ }^{1-3}$ we fully agree with Drs Manzoor and Runcie's conclusions that a long-standing serum folate deficiency could induce neurological and mental disorders, but we have to stress the need for more complete investigations in such cases. CSF folate determinations are useful because a low serum folic acid concentration could induce minor neurological and mental symptoms, while a low or borderline CSF folate in addition could induce major neurological symptoms in spite of the presence of a normal erythrocyte folate level and in the absence of frank anaemia. ${ }^{2} 3$

(3) I suggest the following precautions with respect to folate therapy. (a) We have confirmed a previous report ${ }^{4}$ that after 3-6 months of folic acid therapy there is a progressive fall of serum vitamin $\mathrm{B}_{12}$ in some patients. In practice, after six months of folic acid therapy multivitamin supplements including vitamin $\mathbf{B}_{12}$ have to be added. ${ }^{3}$ (b) High oral or even pharmacological doses of folic acid must be prescribed with great caution for epileptic patients. ${ }^{5}(c)$ In patients from whom carcinomas have been removed or who may have occult metaplasias folic acid administration in maintenance doses must also be limited because of its known stimulation of RNA and DNA metabolism and of cellular division. In such cases folic acid should be administered for 2-3 weeks only and then replaced by vitamin $B_{12}$ 